\title{
Identification of Prognostic Biomarkers Among DAAM1, FHOD1,FMN2 and INF2 in Breast Cancer Patients
}

\section{Yin-Hai Dai}

Department of Surgical Oncology Medicine,The second affiliated hospital of Shaanxi University of Chinese medicine,Xianyang ,712000,PR China

\section{Fuping Li ( $\square$ 1627442702@qq.com )}

Department of Clinical Medicine,Shaanxi University of Chinese Medicine,Xianyang ,712000,PR China https://orcid.org/0000-0003-2138-3330

\section{Wei-Jie Kong}

Department of Clinical Medicine,Shaanxi University of Chinese Medicine,Xianyang ,712000,PR China

\section{Xue-Qin Zhang}

Jincheng institute of Sichuan University,Chengdu ,610000,PR China

\section{Mao Wang}

Department of Surgical Oncology Medicine,The second affiliated hospital of Shaanxi University of Chinese medicine,Xianyang ,712000,PR China

\section{Hai-Long Ma}

Department of Surgical Oncology Medicine,The second affiliated hospital of Shaanxi University of Chinese medicine,Xianyang ,712000,PR China

\section{Qi Wang}

Department of Surgical Oncology Medicine,The second affiliated hospital of Shaanxi University of Chinese medicine,Xianyang ,712000,PR China

\section{Research}

Keywords: breast cancer, formin protein, biomarker, prognosis,Oncomine, Kaplan-Meier plotter

Posted Date: July 28th, 2021

DOI: https://doi.org/10.21203/rs.3.rs-742870/v1

License: (1) This work is licensed under a Creative Commons Attribution 4.0 International License. Read Full License 


\title{
Identification of prognostic biomarkers among DAAM1, FHOD1,FMN2 and INF2 in breast cancer patients
}

\author{
Yin-Hai Dai ${ }^{1, b}$, Fu-Ping Li ${ }^{1, a}$,Wei-Jie Kong ${ }^{1, a}$,Xue-Qin Zhang1,c,Mao Wang ${ }^{2, b}$, \\ Hai-Long Ma ${ }^{2, b}$, Qi Wang ${ }^{2, b}$
}

a Department of Clinical Medicine,Shaanxi University of Chinese Medicine,Xianyang ,712000,PR China

${ }^{b}$ Department of Surgical Oncology Medicine, The second affiliated hospital of Shaanxi University of Chinese medicine,Xianyang ,712000,PR China

'Jincheng institute of Sichuan University,Chengdu ,610000,PR China

Correspondence to:Fu-Ping Li;email:: 1627442702@qq.com,ORCID:0000-0003-2138-3330

Number of words:4399

Number of Figures:4

Number of Table: 1

\section{ABSTRACT}

Background:The formin family proteins are main regulators of actin filaments, which play a crucial role in the migration of cells and carcinogenesis. The specific functions of the formin family proteins in breast cancer still remain unknown.To dissolve this problem, we selected four formin proteins including DAAM1,FHOD1, FMN2 and INF2 and investigated their mRNA expression and survival data in $\mathrm{BC}($ breast carcinoma) patients using diverse databases.

Methods:we used these databases including Oncomine, Ualcan, GEPIA 2, Human Protein Atlas,Metascape,Kaplan-Meier plotter,cBioPortal and TIMER and the software of Cytoscape in our study.

Results:DAAM1 and FMN2 were lowly expressed in BC tissues, while FHOD1 and INF2 were highly expressed in BC tissues. The expression levels of DAAM1, FMN2 and FHOD1 were relevant to major subclasses, and the mRNA level of FHOD1 was related to cancer staging. Moreover,High mRNA levels of FHOD1 
and INF2 were relevant to poorer prognosis of BC patients, while low mRNA level of DAAM1 was correlated with better prognosis.we also found that there were significant associations between the expressions of DAAM1,FHOD1,FMN2 and INF2 and six types of infiltrated immune cells(B Cells, CD $4^{+} \mathrm{T}$ cells, CD ${ }^{+} \mathrm{T}$ cells, neutrophil,macrophage, and dendritic cell).

Conclusions:our study indicated that FHOD1 and INF2 were potential biomarkers to identify short survival of BC patients,FMN2 was potential prognostic marker to suggest favorable survival of BC patients.

Keyword: breast cancer,formin protein,biomarker,prognosis,Oncomine,Kaplan-Meier plotter

\section{INTRODUCTION}

Breast cancer is one of the most common cancers, which seriously threatens the females health.According to the global cancer data survey in 2020 , the incidence of breast cancer has exceeded that of lung cancer, ranking first among female cancers.In 2020, almost 685,000 people died of breast cancer in the world and 2.3 million new cases of breast cancer were diagnosed,the mortality of breast cancer accounted for $15 \%$ of all cancer-related mortality[1].Based on both molecular and histological evidences, breast cancer is divided into four groups, generally referred to luminal A,luminal B,basal-like subtypes and HER2 overexpression[2].At present,the treatments of breast cancer continue to evolve,patients with ER-or PR-or HER2-positive have been greatly benefited from endocrine therapy or molecule-targeting therapy[3].Even though the therapies of breast cancer are developing and improving,including earlier diagnosis and more useful therapeutic methods in the previous decades, it appears to poor prognosis and survival in some special types of BC such as triple-negative breast cancer (TNBC), which ER, PR, and HER2 are all negative.Besides, distal metastasis occurs in 5\%-10\% of breast cancer patients at time of dectection[4].All in all,there are still some inadequacies in present biomarkers that predict prognosis because of melanoma 
heterogeneity, Therefore, further knowledge is needed to discover new diagnostic and prognostic biomarkers, and BC patients can benefit from those.

The formin family proteins are main regulators of actin filaments, which were correlated with the migration of cells and occurrence of different cancers[5]. There are 15 different formin proteins in human tissue, includingFMN1/2,mDia1/2 /3,FHOD1/3,FMNL1/2/3,DAAM1/2,GRD2IP,FHDC1 and INF2 [6].Their common structure is comprised of Formin Homology $1(\mathrm{FH} 1)$ domain and Formin Homology 2(FH2) domain[6].

To date, Only a limited number of studies have proved that the aberrant expressions of some formin family proteins have some prognostic values in human breast cancer.For instance,Jie Mei et al.[7]found that high expression of DAAM1 was significantly correlated with poorer overall survival (OS) of patients with breast cancer.D Heuser et al.[8]found that knockdown of FHOD1 and INF2 lessened the invasion and proliferation of TNBC cells.In addition,A study has showed that the mutation of FMN2 happened in $2 \%$ of the patients who were diagnosed with pure invasive micropapillary carcinoma of the breast[9] .However, the specific functions of the formin family proteins in BC remain unknown. In this study, we aimed to show that the expressions and mutations of DAAM1,FHOD1, FMN2 and INF2 in breast cancer by comprehensive bioinformatical analysis and found potential biomarkers which may have profound significance in the therapy of intractable breast cancer.

\section{MATERIALS AND METHODS}

\section{ONCOMIN database}

ONCOMINE(http://www.oncomine.org), a robust database, an integrated data-ming platform, which provides a powerful set of analysis functions that compute gene expression features,cluster and gene-set modules[10].In our study, data were obtained to assess the expressions of DAAM1, FHOD1,FMN2, and INF2 in breast cancer.Different mRNA expression level were compared using Student's $\mathrm{t}$-test, and the parameters were set as: $\mathrm{P}$ value $=0.001$, fold change $=1.5$, gene rank 
$=10 \%$, and data type $=$ mRNA.

\section{UALCAN}

UALCAN(http://ualcan.path.uab.edu) is a comprehensive online tool, which provides different modules to analyse The Cancer Genome Atlas (TGCA) cancer transcriptome data[11].In this study, the data of mRNA expression were extracted by using the"TGCA Analysis"module and the "breast invasive carcinoma" dataset .Different mRNA expression level were compared using Student's t-test,P value $<0.05$ was deemed significant.

\section{GEPIA 2}

GEPIA 2(http://gepia2.cancer-pku.cn/\#index) is a web-based tool, which provides different modules to analyse normal and cancer gene expression profiling[12].In this study,we explored the associations between the expressions of the four formin proteins and cancer staging by using the "Expression DIY" module of GEPIA 2 , and selected 50 most frequently altered neighboring genes of the four formin proteins via the "Similar Genes Detection"module.Using the "Correlation Analysis"module to analyse the correlation among above four formin proteins.

\section{Human Protein Atlas}

HPA (https://www.proteinatlas.org/) is a freely available database, which contains RNA-sequencing data of 32 different tissues and provides millions of immunohistochemistry images about all major tissues in the huaman body[13].In our study, the immunohistochemistry images were performed for comparison for the protein expression levels of DAAM1,FHOD1 and INF2 between BC tissues and normal tissues.

\section{Kaplan-Meier plotter}

Kaplan-Meier plotter (http://kmplot.com/) is a web-based meta-analysis tool, which is used to evaluate the impact of thousands of genes(mRNA,miRNA, protein) on survival in 21 cancer types [14-15].In our study, to evaluate the effect 
of mRNA expressions of the four formin proteins on survival in breast cancer, we selected OS,RFS,PPS and DMFS as evaluation indicators and performed their survival curves by using Kaplan-Meier plotter,some statistical parameters including hazard ratio(HR),95\% confidence intervals(CIs) and $\mathrm{P}$ values were showed in these survival curves.P value $<0.05$ was deemed significant.

\section{cBioPortal}

CBioPortal (http://www.cbioportal.org/) is a comprehensive online tool which based on TGCA database.Users are able to explore, visualize and analyze cancer transcriptome data by using it[16-17]. In this study,we selected the dataset of Breast Invasive Carcinoma (TGCA,Firehose Legacy) which contained 960 complete samples of 1108 total,and analyzed the genetic alterations of the four formin proteins by this dataset. mRNA expression z-Scores (microarray) with a $\mathrm{z}-$ Score threshold \pm 1.5

\section{Metascape and Cytoscape}

Metascape (https://metascape.org/) is an efficient web-based tool, which provides a comprehensive gene list annotation and analysis function[18].In our study the functions of DAAM1,FHOD1,FMN2, and INF2 and their 50 frequently altered neighboring genes were analyzed by analysis of GO and KEGG via the "Custom Analysis"module.GO enrichment analysis was composed of three parts:biological processes(BP), cellular components(CC) and molecular functions(MF).

Cytoscape (https://cytoscape.org/) is a friendly and convenient software for visualizing molecular interaction network and biological pathways and integrating those networks with annotations[19].In this study, we established PPI network for the four formin proteins and their 50 neighboring genes using it, and screened six hub genes via the "CytoHubba" plugin.Lastly, the picture of PPI was presented in a style of grid layout.

\section{TIMER}

TIMER (https://cistrome.shinyapps.io/timer/) is a intuitive tool which contains a comprehensive source for systematical analysis of immune infiltrates across 
various tumor types[20-21]. In this study,we analyzed the the correlation between the expressions of DAAM1,FHOD1,FMN2, and INF2 and six tpyes of infiltrated immune cells by using "Gene" Moudle.

\section{RESULTS}

\section{mRNA levels of DAAM1,FHOD1,FMN2, and INF2 in BC patients}

We first analyzed the transcriptional levels of DAAM1,FHOD1,FMN2, and INF 2 in $\mathrm{BC}$ and normal breast tissues by using the Oncomine database(http://www. oncomine.org ). The results were shown in Figure 1A and Table 1. the mRNA levels of DAAM1,FHOD1 and FMN2 in BC were significantly decreased, while the expression level of INF2 was significantly increased.In Curtis Breast dataset [22],DAAM1 was decreased in benign breast neoplasm (fold change=-1.648,p= 4.22E-8), invasive breast carcinoma (fold change $=-1.635, \mathrm{p}=4.10 \mathrm{E}-8$ ), mucinous breast carcinoma (fold change $=-1.701, \mathrm{p}=4.43 \mathrm{E}-13$ ), ductal breast carcinoma(fold change $=-1.586, \mathrm{p}=4.22 \mathrm{E}-8)$, breast carcinoma (fold change $=-1.625, \mathrm{p}=4.70 \mathrm{E}-5)$, and medullary breast carcinoma (fold change $=-1.700, \mathrm{p}=3.67 \mathrm{E}-4$ ) versus normal tiss ues .Likewise, Gluck breast statistics showed that DAAM1 was down-regulated in invasive breast carcinoma (fold change $=-1.508, \mathrm{p}=3.67 \mathrm{E}-4$ ) compared to normal breast tissues[23].

Finak breast statistics showed that the expression of FHOD1was lower in invasive breast carcinoma stroma than normal breast tissues (fold chamge= $-6.126, p=6.71 \mathrm{E}-28)[24]$. Ma et al.[25]found that FMN2 was down-regulated in ductal breast carcinoma stroma (fold change $=-2.297, \mathrm{p}=4.93 \mathrm{E}-4$ ) and invasive ductal breast carcinoma stroma(fold change $=-2.300, p=5.90 \mathrm{E}-4$ ) versus normal breast tissues.In Zhao breast statistics, the expression of INF2 was higher in lobular breast carcinoma stroma (fold change $=1.786, \mathrm{p}=2.96 \mathrm{E}-6$ ) and invasive ductal breast carcinoma (fold change $=1.667, \mathrm{p}=2.96 \mathrm{E}-6$ ) compared to normal breast tissues[26]. Ma breast statistics also found that INF2 was up-regulated in invasive ductal breast carcinoma epithelia (fold change $=1.772, p=9.31 \mathrm{E}-5$ ) 
[25].

\section{Linkages of mRNA levels of DAAM1,FHOD1,FMN2, and INF2 with clinicopathological parameters of $\mathrm{BC}$ patients}

We explored the mRNA expressions of DAAM1,FHOD1,FMN2 and INF2 in BC and normal breast samples via the UALCAN database(http://ualcan.path.uab. edu).Different from ONCOMINE database, the expression levels of DAAM1 and FMN2 in breast cancer tissue were significantly down-regulated, while the expression levels of FHOD1 and INF2 were significantly up-regulated (Figure1B).Subsequently, we evaluated the associations between the expressions of the four formin proteins and major subclasses and cancer staging by using the UALCAN database and the GEPIA2 database (http://gepia2.cancer-pku.cn/\#index ).The results showed that the lowest mRNA levels of DAAM1 and FMN2 were found in TNBC and the highest expression level of FHOD1 was discovered in HER2-positive breast cancer among major subclasses (Figure 1D). Moreover, from Figure 1C, we could see that the mRNA level of FHOD1 significantly changed $(\mathrm{p}<0.05)$ in different stage, whereas DAAM1,FMN2, and INF2 did not $($ all $\mathrm{p}>0.05)$.

We also analyzed the immunohistochemisty (IHC) of DAAM1,FHOD1 and INF2 in BC tissues and normal breast tissues by using HPA database (https://www.proteinatlas.org/).Currently, the IHC image of FMN2 was not found in HPA database.The result showed that high or medium expressions of DAAM1,FHOD1, and INF2 were detected in breast cancer tissues, whereas medium or low protein expressionsof them were detected in normal breast tissues(Figure 2A ).

\section{The prognostic value of DAAM1,FHOD1,FMN2, and INF2 in BC patients} we evaluated the prognostic value of DAAM1,FHOD1,FMN2 and INF2 in the 
survival of breast cancer via the online tool of Kaplan-Meier Plotter (http://kmplot.com/). The curves of overall survival(OS), relapse-free survival (RFS),post-progression survival(PPS) and distant metastasis free survival (DMFS) were showed in Figure 2B.High mRNA expressions of FHOD1 and INF2 were relevant to poor OS,PPS and DMFS of BC patients (all $\mathrm{p}<0.05$ ), while low mRNA expression of DAAM1 was relevant to high OS,RFS,PPS and DMFS of BC patients (all $\mathrm{p}<0.05)$. We also found that low mRNA level of FMN2 was related to short RFS of BC patients $(\mathrm{p}<0.05)$. 


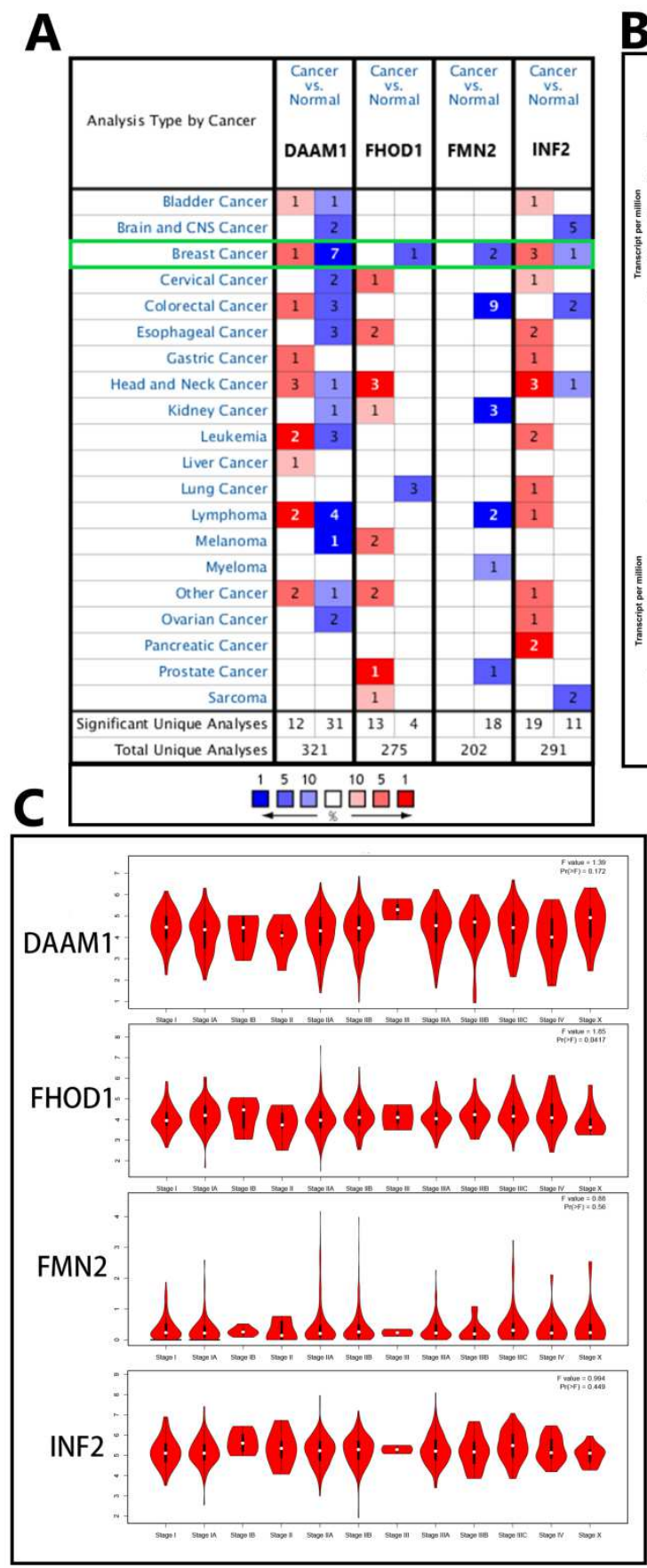

B
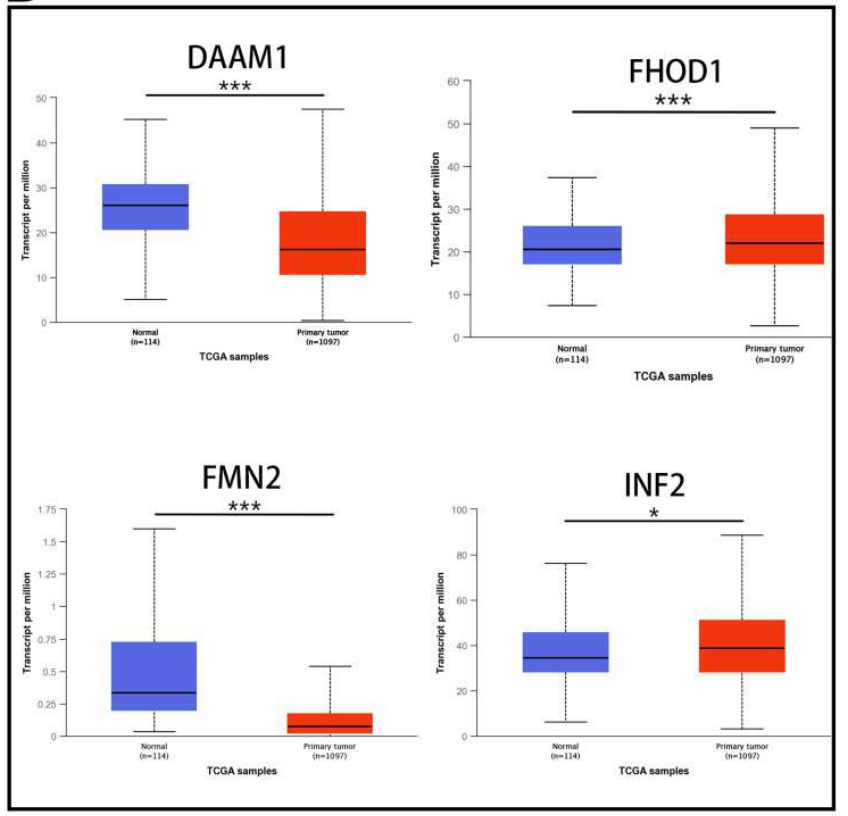

\section{D}

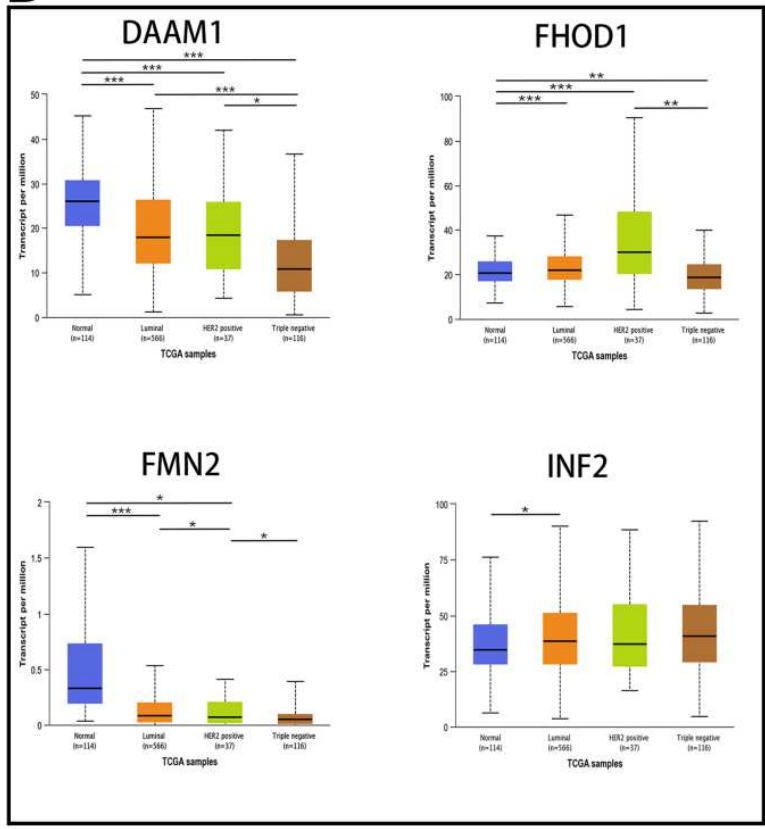

Figure 1. mRNA levels of DAAM1,FHOD1,FMN2 and INF2 and their linkages with clinicopathological parameters of BC patients(ONCOMINE,UALCAN,GEPIA 2).(A)The mRNA levels of DAAM1,FHOD1,FMN2 and INF2 in 20 types of cancers (ONCOMINE).(B)The mRNA levels of DAAM1,FHOD1,FMN2 and INF2 in BC samples and normal breast samples(UALCAN).(C)Relevance between 
the mRNA expressions of DAAM1, FHOD1, FMN2 and INF2 and cancer staging in BC patients (GEPIA2).(D)Association between the mRNA expression of DAAM1,FHOD1,FMN2 and INF2 and major subclasses in breast cancer $(\mathrm{UALCAN}) \cdot{ }^{*} \mathrm{p}<0.05,{ }^{*} \mathrm{p}<0.01, * * * \mathrm{p}<0.001$

\section{Genetic mutations and co-expression of DAAM1,FHOD1,FMN2 and INF2 in patients with breast cancer}

Genetic mutations and co-expression of the four formin proteins were analyzed via the cBioPortal database(http://www.cbioportal.org/). The results were showed in Figure 2C,D and Figure 3A .In total,37\%(352/960) of patients with breast cancer had at least two genetic mutations.DAAM1,FHOD1,FMN2 and INF2 were altered in $11 \%, 7 \%, 18 \%$ and $9 \%$ of the 960 samples (Figure 2C). Similarly, the degree of genetic mutations of the four formin proteins was presented in the Expression Heatmap(Figure 2D), Moreover, we analyzed the correlations among the four formin proteins via the GEPIA2 database.As was showed in Figure 3A,there was meaningful and positive associations between FHOD1 and INF2 $(\mathrm{r}=0.38, \mathrm{p}<0.05)$. 
Table 1. Significant changes of the expression of DAAM1,FHOD1,FMN2 and

INF2 in mRNA level between BC and normal breast tissues(ONCOMINE)

\begin{tabular}{llllll}
\hline & $\begin{array}{l}\text { Types of breast cancer } \\
\text { normal Breast Tissue }\end{array}$ & $\begin{array}{l}\text { Fold } \\
\text { Change }\end{array}$ & P value & T-test & Ref \\
\hline DAAM1 & benign breast neoplsm & -1.648 & $4.22 \mathrm{E}-8$ & -11.581 & Cortis Breast[22] \\
& invasive breast carcinoma & -1.635 & $4.10 \mathrm{E}-8$ & -7.311 & Cortis Breast [22] \\
& mucinous breast carcinoma & -1.701 & $4.43 \mathrm{E}-13$ & -8.885 & Cortis Breast [22] \\
& ductal breast carcinoma & -1.586 & $2.80 \mathrm{E}-4$ & -4.903 & Cortis Breast [22] \\
& breast carcinoma & -1.625 & $4.70 \mathrm{E}-5$ & -5.090 & Cortis Breast [22] \\
& medullary breast carcinoma & -1.700 & $1.38 \mathrm{E}-8$ & -6.946 & Cortis Breast [22] \\
& invasive breast carcinoma & -1.508 & $3.67 \mathrm{E}-4$ & -6.520 & Gluck Breast [23] \\
FHOD1 & invasive breast carcinoma stroma & -6.126 & $6.71 \mathrm{E}-28$ & -25.091 & Finak Breast[24] \\
& & & & & \\
\hline
\end{tabular}




\begin{tabular}{llllll}
\hline FMN2 & ductal breast carcinoma stoma & -2.297 & $4.93 \mathrm{E}-4$ & -3.891 & Ma Breast[25] \\
& $\begin{array}{l}\text { invasive ductal breast carcinoma } \\
\text { INF2 }\end{array}$ & -2.300 & $5.90 \mathrm{E}-4$ & -3.792 & Ma Breast[25] \\
& $\begin{array}{l}\text { invasive ductal breast carcinoma } \\
\text { lobular breast carcinoma }\end{array}$ & 1.667 & $2.78 \mathrm{E}-6$ & 7.117 & Zhao Breast[26] \\
& $\begin{array}{l}\text { invasive ductal breast carcinoma } \\
\text { epithelia }\end{array}$ & 1.772 & $9.31 \mathrm{E}-5$ & 5.026 & Ma Breast[25] \\
& & & & \\
\hline
\end{tabular}



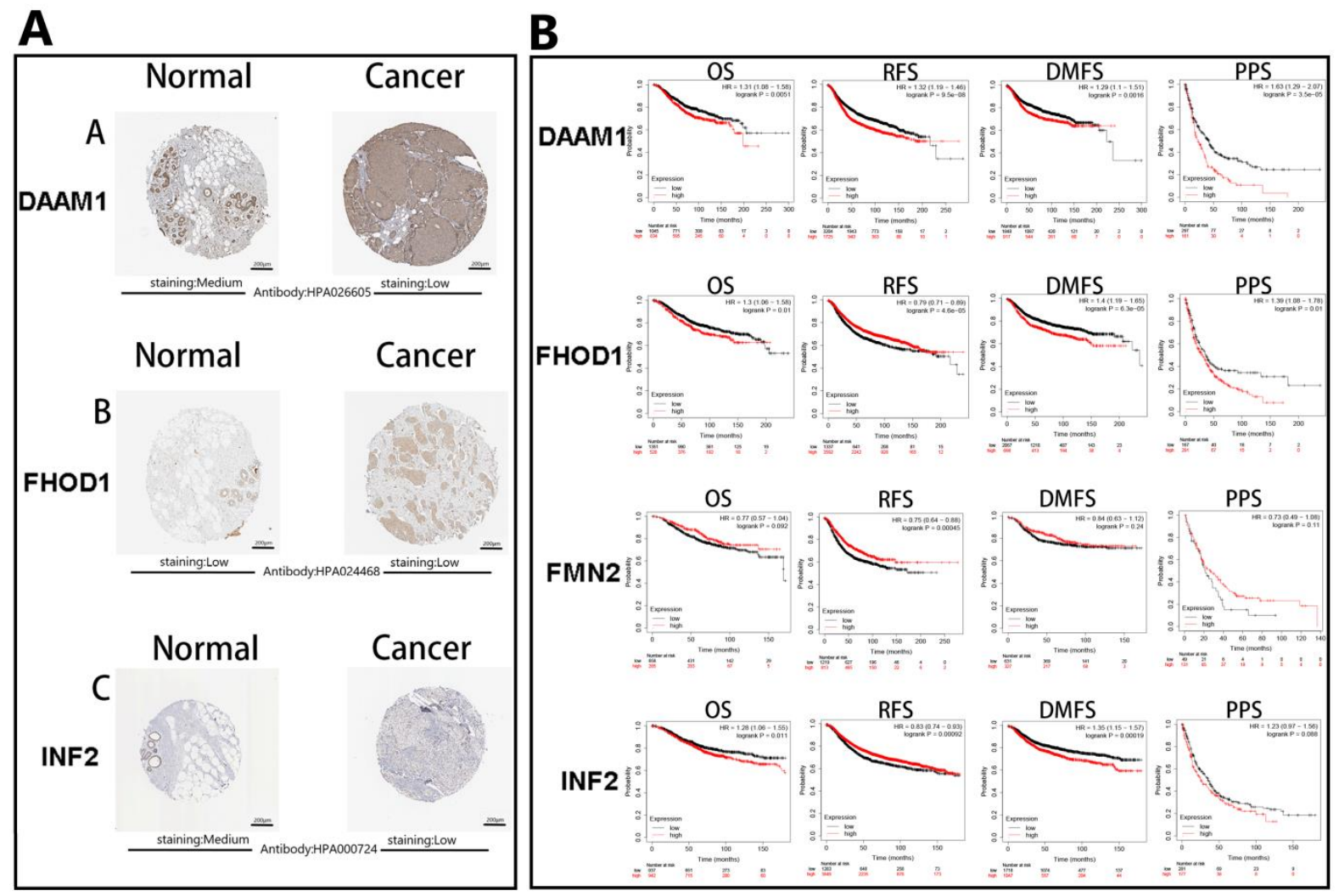

C

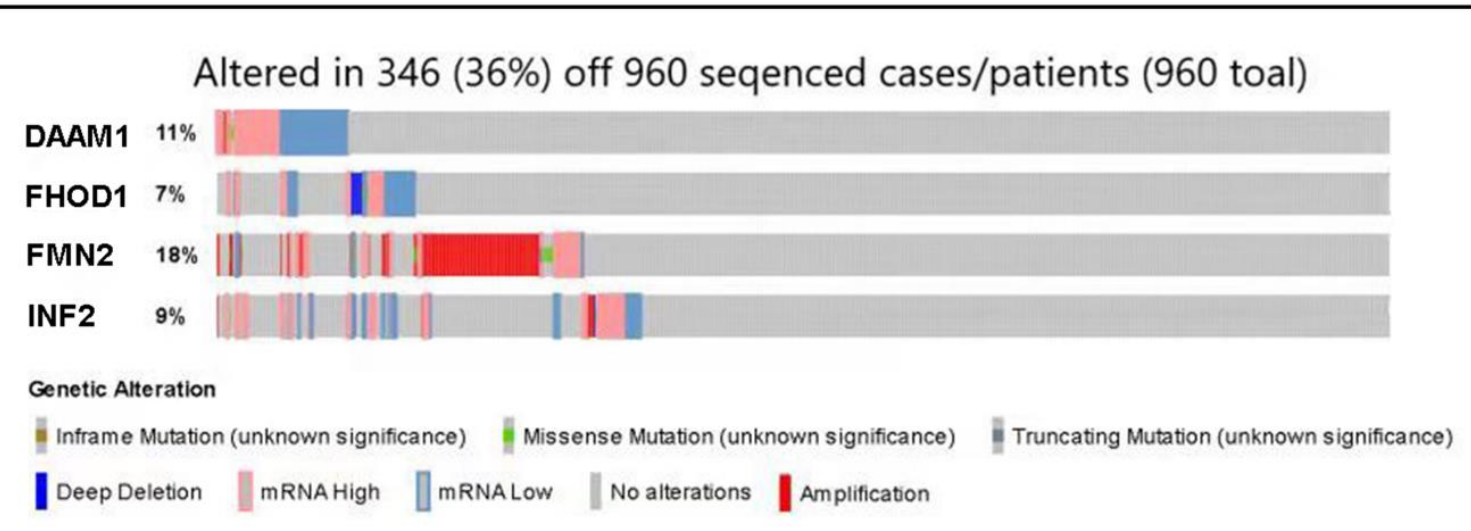

D

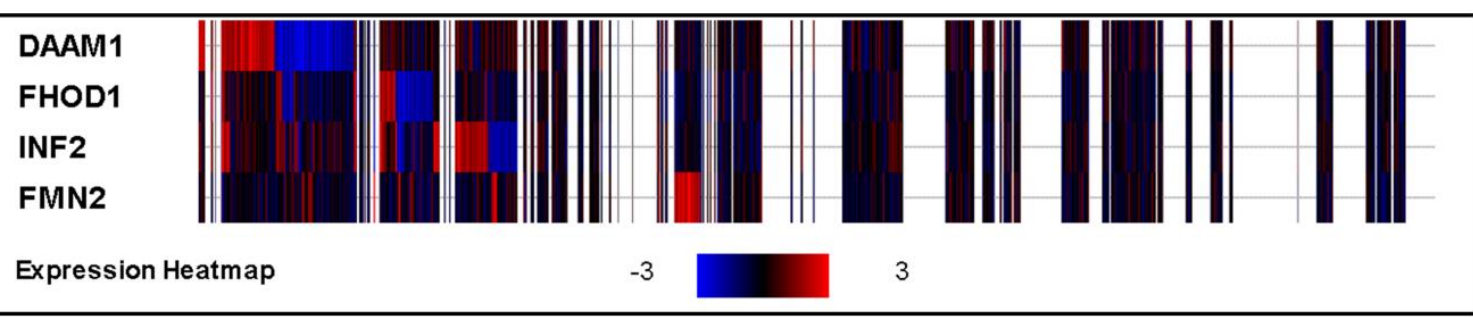

Figure 2. Immunohistochemisty (IHC), Prognostic value, and Genetic mutations of DAAM1,FHOD1,FMN2 and INF2 in breast cancer(HPA, Kaplan-Meier Plotter,cBioPortal).(A)The IHC images about the expressions of DAAM1,FHOD 
1 and INF2 in breast cancer and normal breast tissues(HPA).(B) The prognostic values of DAAM1,FHOD1,FMN2 and INF2 in BC patients in OS, RFS,DMFS and PPS curves(Kaplan-Meier Plotter).(C,D) Genetic mutations and expression heatmap of DAAM1 of FHOD1,FMN2 and INF2 in patients with breast cancer (cBioPortal).

\section{Established protein-protein interaction (PPI) network and analyzed the} functions and pathways of DAAM1,FHOD1,FMN2, and INF2 in BC patients

Firstly,we selected 50 most frequently altered neighboring genes of the four formin proteins by using the GEPIA2 database.And then,we established PPI network for the four formin proteins and their 50 frequently neighboring genes of by using the software of Cytoscape(https://cytoscape.org/). The result was showed in Figure 3B,six hub genes including RP11-542A14.2,RNY1P4,KRT8P13, TGFB1, FASTK, and MICALL2 were tightly related to the alterations of DAAM1,FHOD1,FMN2, and INF2.

Next, we predicted the functions of DAAM1,FHOD1, FMN2, and INF2 and their 50 frequently altered neighboring genes by analysis of GO and KEGG using Metascape (https://metascape.org/). The GO enrichment analysis showed GO:0043 61(proteasome-mediated ubiqutin-dependent protein catabolic process), GO:0001 503(ossification), GO:0043691(reverse cholesterol transport), GO:0032963(collagen metabolic process),GO:0031093(platelet alpha gromule lumen), GO:0098687 (chromosomal region),GO:0017653(transcription,repress complex), and GO:0001 103 (RNA polymerase II repressing transcription factor binding) were meaningful modulated by alterations of the four formin proteins (Figure 3C). Moreover, KEGG pathway enrichment analysis indicated that hsa:05213(Endometrial cancer) ,ko:05152 (Tuberculosis) and ko:04450 (signaling pathways regulating piuripotency of stem cells) were significantly related to alterations of the four formin proteins(Figure 3D). 

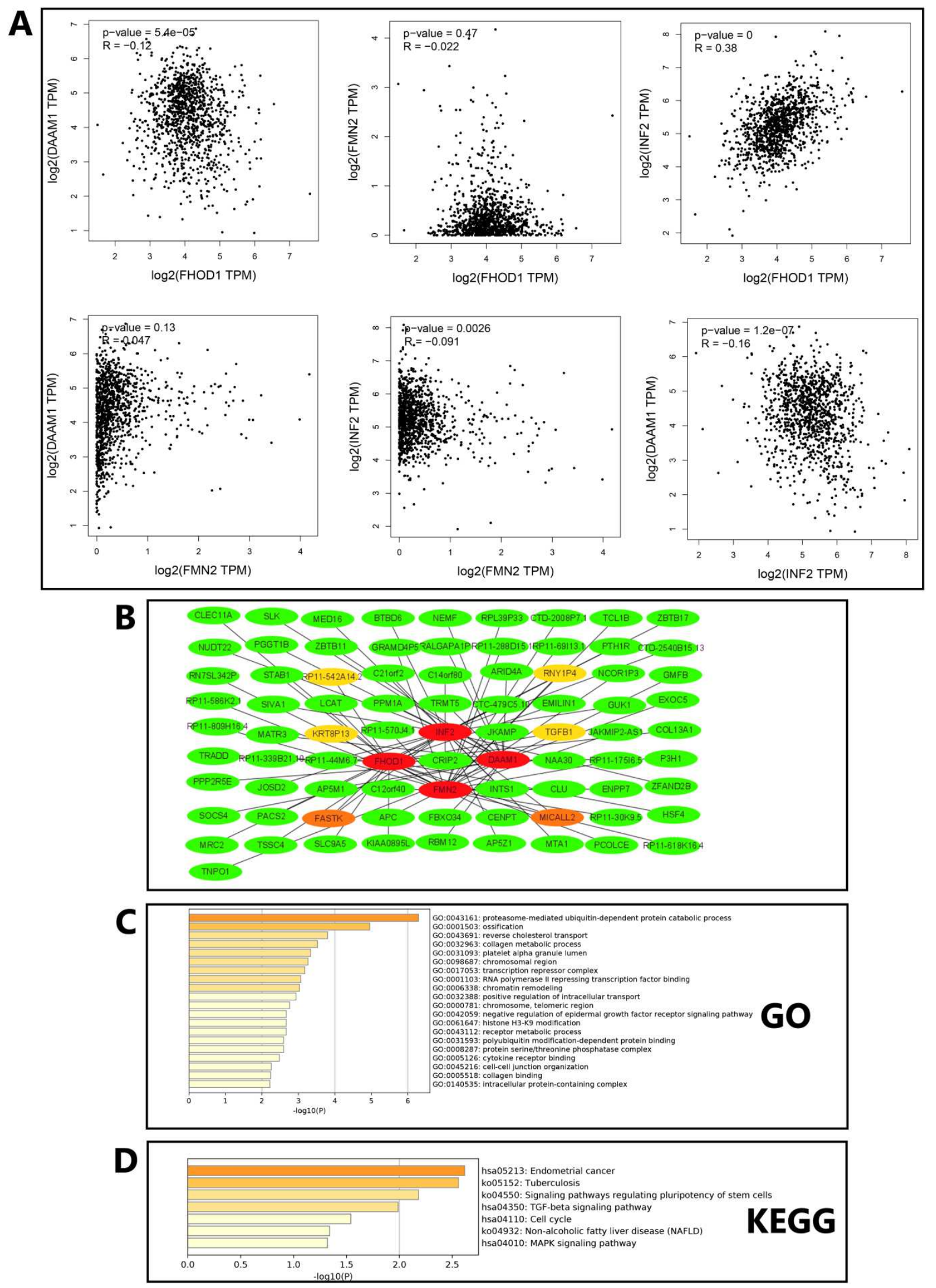
Figure 3 .The correlation among the four formin proteins, PPI network and functional and enrichment analysis for DAAM1,FHOD1,FMN2, and INF2 and their 50 frequently altered neighboring genes in breast cancer ( GPEIA 2, Cytoscape and Metascape) .(A) The correlation among the four formin proteins (GEPIA 2). (B)The PPI network between the four formin proteins and their 50 frequently altered neighboring genes(Cytoscape)(C) GO enrichment analysis for the four formin proteins and their 50 frequently altered neighboring genes(Metascape).(D)KEGG pathway enrichment analysis for the four formin proteins and their 50 frequently altered neighboring genes(Metascape).

\section{Immune Cell Infiltration of DAAM1,FHOD1,FMN2, and INF2 in BC patients}

Lastly, we attempted to analyse the correlation betweenDAAM1,FHOD1,FMN2 and INF2 and infiltrated immune cells by using the TIMER database (https://cistrome.shinyapps.io/timer/). The results were showed in Figure 4. DAAM1 expression was positively correlated with the infiltration of $\mathrm{CD}^{+} \mathrm{T}$ cells $(\mathrm{Cor}=0.324, \mathrm{p}=2.74 \mathrm{E}-25), \mathrm{CD} 4^{+} \mathrm{T}$ cells $(\mathrm{Cor}=0.111, \mathrm{p}=5.72 \mathrm{E}-4)$, macrophage $(\mathrm{Cor}=0$. $271, \mathrm{p}=4.89 \mathrm{E}-18)$, neutrophil $(\mathrm{Cor}=0.165, \mathrm{p}=2.92 \mathrm{E}-7)$ and dendritic cell $(\mathrm{Cor}=0.106$, $\mathrm{p}=1.04 \mathrm{E}-31)\left(\right.$ Figure 4A).FHOD1 expression was inversely correlated with $\mathrm{CD}^{+} \mathrm{T}$ cells $($ Cor $=-0.128, p=6.34 \mathrm{E}-5)$ and positively correlated with infiltration of $\mathrm{CD}^{+}{ }^{+} \mathrm{T}$ cells $(\operatorname{Cor}=0.088, \mathrm{p}=6.16 \mathrm{E}-3)$ and macrophage $(\mathrm{Cor}=0.077, \mathrm{P}=1.54 \mathrm{E}-2)($ Figure $4 \mathrm{~B})$. FMN2 was positively associated with CD8+T cells (Cor=0.179,p=1.78E-8),CD4 ${ }^{+}$ $\mathrm{T}$ cells $(\mathrm{Cor}=0.112, \mathrm{p}=4.95 \mathrm{E}-4)$, macrophage $(\mathrm{Cor}=0.245, \mathrm{p}=7.12 \mathrm{E}-15)$, , neutrophil $(\operatorname{Cor}=0.104, \mathrm{p}=1.35 \mathrm{E}-3)$ and dendritic cell $(\mathrm{Cor}=0.103, \mathrm{p}=1.55 \mathrm{E}-3) \quad$ (Figure 4C). INF2 expression was negatively correlated with infiltration of $\mathrm{CD}^{+} \mathrm{T}$ cells $(\mathrm{Cor}=-0.17, \mathrm{p}=8.72 \mathrm{E}-8)$ and macrophage $(\mathrm{Cor}=-0.088, \mathrm{p}=5.98 \mathrm{E}-3)$ and positively related to $\mathrm{CD} 4+\mathrm{T}$ cells $(\mathrm{Cor}=0.146, \mathrm{p}=5.25 \mathrm{E}-6)($ Figure $4 \mathrm{D})$. 
A

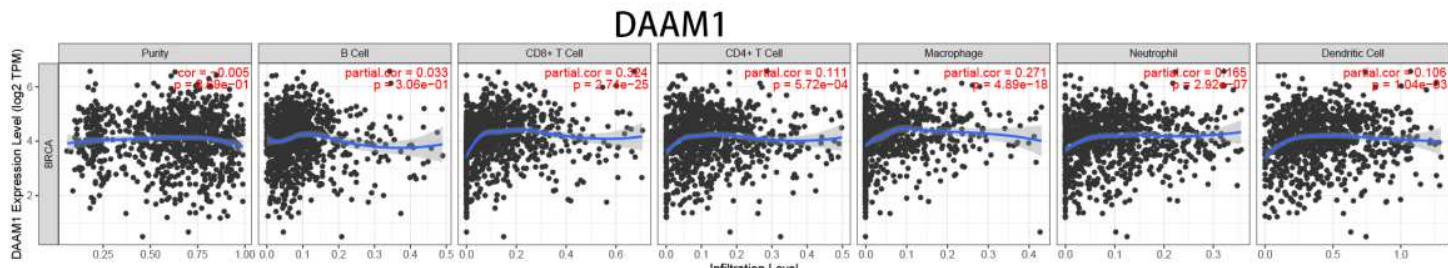

B

FHOD1

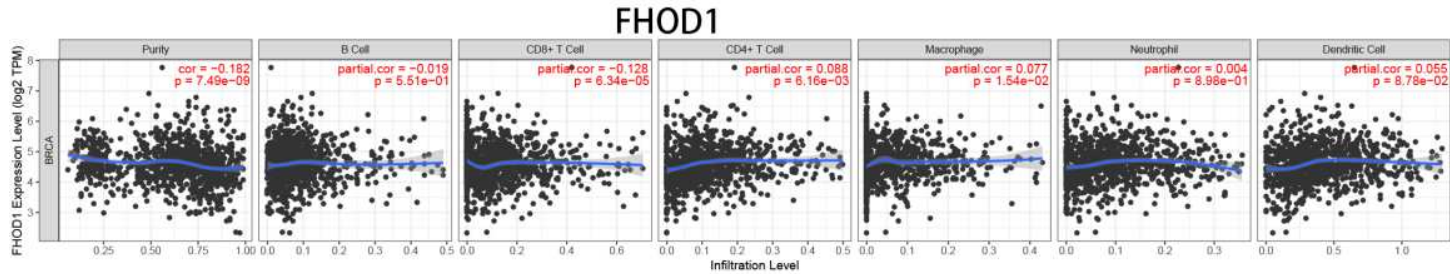

C

FMN2

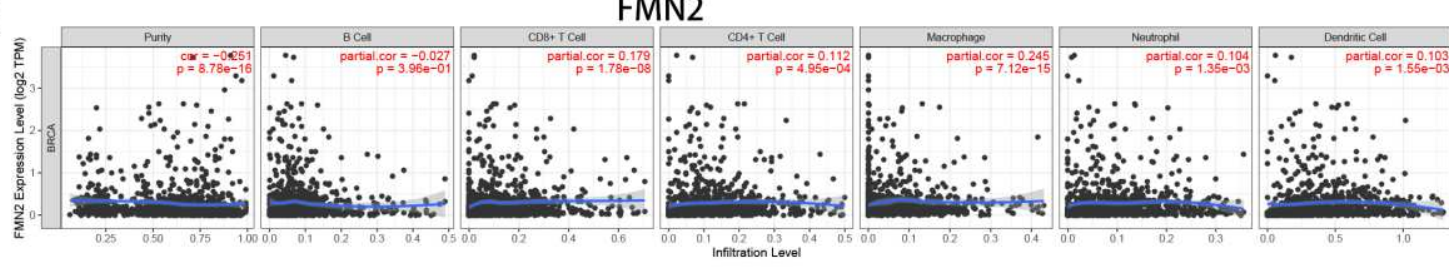

D

INF2

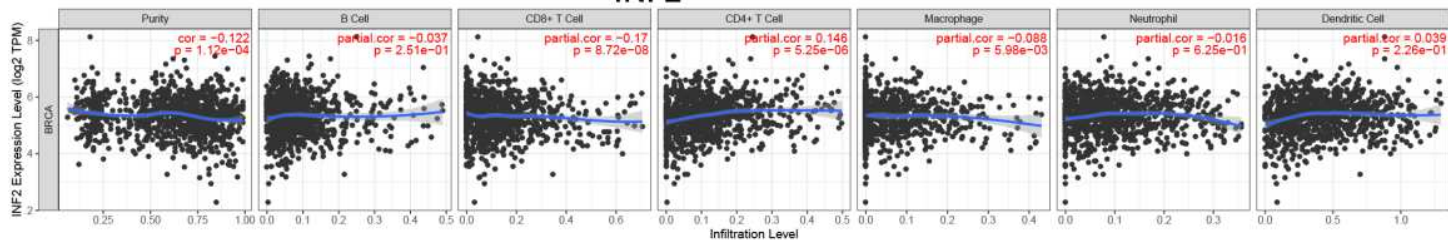

\section{Figure 4.The association between DAAM1,FHOD1,FMN2, and INF2 and six types of infiltrated immune cells(TIMER).}

\section{DISCUSSION}

Formin proteins were initially detected in mice and the mutations of them resulted in aberrant limb development[27] .In addition to, they play an important role in the migration of cells and the onset of cancers[5]. A few of prior studies indicated that the formin proteins were associated with several human cancers including gastric cancer[28,29], colorectal cancer[30], melanomas [31],glioblastomma[32] and breast carcinoma[7].However, the specific mechanisms of the formin family proteins in breast cancer have not been completely illuminated.In this paper,we selected four formin proteins including DAAM1, FHOD1, FMN2 and INF2, and explored their mRNA expressions and prognostic values in breast 
carcinoma. Of course, we hope our findings will provide useful assistance for the diagnosis and treatment of breast cancer.

DAAM1 was shown to be a target gene of miR-613, and miR-613 inhibited the migration and invasion of TNBC cells by downregulating the expression of DAAM1[33].Jie Mei et al.[7] found that overexpression of DAAM1 was significantly related to poorer OS of BC patients. Inconsistenly, In our findings, we observed that DAAM1 played a conflict role in breast cancer,on one hand, DAAM1 was lower expressed in breast carcinoma than normal breast tissues, and the lowest expression level of DAAM1 was discovered in TNBC among major subclassess,but mRNA expression of DAAM1 was not significantly relevant to cancer staging. However, on the other hand,low mRNA level of DAAM1 was relevant to high OS,RFS,PPS, and DMFS.Therefore, it is still indispensable to develop further research to clarify the precise role of DAAM1 in BC.

FHOD1 mediates the migration and proliferation of several malignancies, including breast cancer [8,34].M Peippo et al.[35]found that knockdown of FHOD1 in melanoma cells impaired migratory capacity of these cells.Micro RNA-200c inhibits the invasion and migration of $\mathrm{BC}$ cells by targeting FHOD1[34].In this study, the mRNA and protein expression of FHOD1 was higher in BC than normal breast tissues, the mRNA level of FHOD1 significantly changed in different stage, and the highest expression level of FHOD1 was discovered in HER2-positive breast cancer among major subclassess.In addition to, High mRNA levels of FHOD1 were relevant to poor OS,PPS, and DMFS .Interesting,FHOD1 expression was positively correlated with the infiltration $\mathrm{CD}^{+} \mathrm{T}$ cells and macrophage.By taking above findings into account,our study revealed that FHOD1 played an oncogenic role in breast cancer.

FMN2 dysregulation has been found in several carcinomas[9,36,37].Report from Li et al showed that the downregulation of FMN2 promoter methylation played a crucial role in early colorectal carcinogensis[36].FHOD1 was shown to 
be a downstream gene of miR-144, and the downregulation of miR-144 was associated with human acute lymophoblastic leukemia(ALL)[37].Our study showed that the mRNA expression of FMN2 was lower in BC than normal breast tissues, and the lowest FHOD1 expression was found in HER2-positive breast cancer among major subclassess, while its expression had no correlation with patients'individual cancer staging. Interestingly, low mRNA level of FMN2 was relevant to poor RFS.Furthermore,FMN2 expression was positively related to the infiltration of $\mathrm{CD} 8+\mathrm{T}$ cells, $\mathrm{CD} 4^{+} \mathrm{T}$ cells, macrophage, neutrophi and dendritic celll.Taken together, FMN2 appeared to serve as a tumor suppressor in BC.

Similar to FHOD1, High expression of INF2 is correlated with several cancers $[8,38]$.SPOP (speckle-type POZ protein) was a mutated gene in prostate cancer,which increased the expression of INF2, and overexpression of INF2 played a important role in prostate cancer cell migration and invasion[38].D Heuser et al.[8]found that knockdown of INF2 lessened the invasion and proliferation of TNBC cells.Likewise, In our report, INF2 was higher mRNA expressed in breast carcinoma than normal breast, but there were no significant correlations between the major subclassess and cancer staging.In addition, High expression level of INF2 was relevant to poor OS,PPS and DMFS. Moreover,INF2 expression was negatively correlated with infiltration of $\mathrm{CD}^{+} \mathrm{T}$ cells and macrophage.Taken together,It seemed to indicate that INF2 played an oncogenic role in breast cancer.

In our study,there were some limitations should be acknowledged,Firstly, we did not analyse all formin proteins systematically. Secondly, we did not explore underlying mechanisms between the above-mentioned four formin proteins and breast cancer.Thirdly, the analysis between mRNA level and immune cell inflitration can reflect a few aspects of immune status,but not all,patients with breast cancer.Moreover,there are still more large-scale and multi-centre clinical studies to be conducted to validate our findings.

\section{Conclusions}


In conclusion, FHOD1 and INF2 were potential biomarkers to identify short survival of BC patients, and FMN2 was potential prognostic marker to suggest favorable survival of $\mathrm{BC}$ patients. Therefore, the above findings may offer us a new therapeutic direction of FHOD1,FMN2 and INF2 in breast cancer.

\section{ABBREVIATIONS}

ER:estrogen receptor;PR:progesterone receptor;HER-2:human epidermal growth factor receptor-2;DAAM1:disheveled-associated activator of morphgenesis1, FHOD1:formin homology1;FMN2:formin 2;INF2:inverted formin2;GO:gene ontology;KEGG:kyoto encyclopedia of gens and genomes.

\section{DECLARATIONS:}

\section{Ethic approval and consent to participate}

Not applicable

\section{Consent for publication}

Not applicable

\section{Availability of data and materials}

The datasets used during the current study are available from the corresponding author on reasonable request

\section{Author contributions}

FL and $\mathrm{XZ}$ wrote manuscript,WK,MW,HM and QW assisted in analysis work,FL and YD designed the study. The authors all approved of the final manuscript to be submitted to "Journal of Biological Research-Thessalanki".

\section{Conflicts of interest}

The authors declare no competing interests

\section{Funding}

This study was supported by the Special Scientific research project of Education Department of Shaanxi Province(grant number:17JK0209), the discipline innovation-team project of Shaanxi university of Chinese Medicine(grant number :2 019-SY03). 


\section{Acknowledgements}

Not applicable

\section{REFERERCES}

[1] Siegel R L, Miller K D, Fuchs H E, et al. Cancer Statistics, 2021[J]. CA Cancer J Clin, 2021,71(1):7-33.

[2] Yeo S K, Guan J L. Breast Cancer: Multiple Subtypes within a Tumor?[J].Trends Cancer,2017,3 (11):753-760.

[3] Waks A G, Winer E P. Breast Cancer Treatment: A Review[J]. JAMA, 2019,321(3):288-300.

[4] Cardoso F, Fallowfield L, Costa A, et al. Locally recurrent or metastatic breast cancer: ESMO Clinical Practice Guidelines for diagnosis, treatment and follow-up[J]. Ann Oncol, 2011,22 Suppl 6:i25-i30.

[5] Chesarone M A, DuPage A G, Goode B L. Unleashing formins to remodel the actin and microtubule cytoskeletons[J]. Nat Rev Mol Cell Biol, 2010,11(1):62-74.

[6] Schönichen A, Geyer M. Fifteen formins for an actin filament: a molecular view on the regulation of human formins[J]. Biochim Biophys Acta, 2010,1803(2):152-163.

[7] Mei J, Xu B, Hao L, et al. Overexpressed DAAM1 correlates with metastasis and predicts poor prognosis in breast cancer[J]. Pathol Res Pract, 2020,216(3):152736.

[8] Heuser V D, Mansuri N, Mogg J, et al. Formin Proteins FHOD1 and INF2 in Triple-Negative Breast Cancer: Association With Basal Markers and Functional Activities[J].Breast Cancer (Auckl),2018,12:2017 4617545 .

[9] Gruel N, Benhamo V, Bhalshankar J, et al. Polarity gene alterations in pure invasive micropapillary carcinomas of the breast[J]. Breast Cancer Res, 2014,16(3):R46.

[10] Rhodes D R, Yu J, Shanker K, et al. ONCOMINE: a cancer microarray database and integrated data-mining platform[J]. Neoplasia, 2004,6(1):1-6.

[11] Chandrashekar D S, Bashel B, Balasubramanya S, et al. UALCAN: A Portal for Facilitating Tumor Subgroup Gene Expression and Survival Analyses[J]. Neoplasia, 2017,19(8):649-658.

[12] Tang Z, Li C, Kang B, et al. GEPIA: a web server for cancer and normal gene expression profiling and interactive analyses[J]. Nucleic Acids Res, 2017,45(W1):W98-W102.

[13] Uhlén M, Fagerberg L, Hallström B M, et al. Proteomics. Tissue-based map of the human proteome[J]. 
Science, 2015,347(6220):1260419.

[14] Nagy Á, Munkácsy G, Györffy B. Pancancer survival analysis of cancer hallmark genes[J]. Sci Rep, 2021,11(1):6047.

[15] Györffy B, Surowiak P, Budczies J, et al. Online survival analysis software to assess the prognostic value of biomarkers using transcriptomic data in non-small-cell lung cancer[J]. PLoS One, 2013,8(12):e82241.

[16] Cerami E, Gao J, Dogrusoz U, et al. The cBio cancer genomics portal: an open platform for exploring multidimensional cancer genomics data[J]. Cancer Discov, 2012,2(5):401-404.

[17] Gao J, Aksoy B A, Dogrusoz U, et al. Integrative analysis of complex cancer genomics and clinical profiles using the cBioPortal[J]. Sci Signal, 2013,6(269):11.

[18] Zhou Y, Zhou B, Pache L, et al. Metascape provides a biologist-oriented resource for the analysis of systems-level datasets[J]. Nat Commun, 2019,10(1):1523.

[19] Otasek D, Morris J H, Bouças J, et al. Cytoscape Automation: empowering workflow-based network analysis[J]. Genome Biol, 2019,20(1):185.

[20] Li T, Fan J, Wang B, et al. TIMER: A Web Server for Comprehensive Analysis of Tumor-Infiltrating Immune Cells[J]. Cancer Res, 2017,77(21):e108-e110.

[21] Li B, Severson E, Pignon J C, et al. Comprehensive analyses of tumor immunity: implications for cancer immunotherapy[J]. Genome Biol, 2016,17(1):174.

[22] Curtis C, Shah S P, Chin S F, et al. The genomic and transcriptomic architecture of 2,000 breast tumours reveals novel subgroups[J]. Nature, 2012,486(7403):346-352.

[23] Glück S, Ross J S, Royce M, et al. TP53 genomics predict higher clinical and pathologic tumor response in operable early-stage breast cancer treated with docetaxel-capecitabine \pm trastuzumab $[\mathrm{J}]$. Breast Cancer Res Treat, 2012,132(3):781-791.

[24] Finak G, Bertos N, Pepin F, et al. Stromal gene expression predicts clinical outcome in breast cancer[J]. Nat Med, 2008,14(5):518-527.

[25] Ma X J, Dahiya S, Richardson E, et al. Gene expression profiling of the tumor microenvironment during breast cancer progression[J]. Breast Cancer Res, 2009,11(1):R7.

[26] Zhao H, Langerød A, Ji Y, et al. Different gene expression patterns in invasive lobular and ductal carcinomas of the breast[J]. Mol Biol Cell, 2004,15(6):2523-2536.

[27] Woychik R P, Maas R L, Zeller R, et al. 'Formins': proteins deduced from the alternative transcripts of the limb deformity gene[J]. Nature, 1990,346(6287):850-853.

[28] Zhong B, Wang K, Xu H, et al. Silencing Formin-like 2 inhibits growth and metastasis of gastric cancer cells through suppressing internalization of integrins[J]. Cancer Cell Int, 2018,18:79.

[29] Mansuri N, Heuser V D, Birkman E M, et al. FHOD1 and FMNL1 formin proteins in intestinal gastric cancer: correlation with tumor-infiltrating T lymphocytes and molecular subtypes[J]. Gastric Cancer, 2021.

[30] Shao H J, Li Q, Shi T, et al. LINC00707 promotes cell proliferation and invasion of colorectal cancer via miR-206/FMNL2 axis[J]. Eur Rev Med Pharmacol Sci, 2019,23(9):3749-3759.

[31] Peippo M, Gardberg M, Lamminen T, et al. FHOD1 formin is upregulated in melanomas and modifies proliferation and tumor growth[J]. Exp Cell Res, 2017,350(1):267-278.

[32] Higa N, Shinsato Y, Kamil M, et al. Formin-like 1 (FMNL1) Is Associated with Glioblastoma Multiforme Mesenchymal Subtype and Independently Predicts Poor Prognosis[J]. Int J Mol Sci, 2019,20(24).

[33] Xiong H, Yan T, Zhang W, et al. miR-613 inhibits cell migration and invasion by downregulating Daam1 in triple-negative breast cancer[J]. Cell Signal, 2018,44:33-42.

[34] Jurmeister S, Baumann M, Balwierz A, et al. MicroRNA-200c represses migration and invasion of breast cancer cells by targeting actin-regulatory proteins FHOD1 and PPM1F[J]. Mol Cell Biol,2012,32 
(3):633-651.

[35] Peippo M, Gardberg M, Lamminen T, et al. FHOD1 formin is upregulated in melanomas and modifies proliferation and tumor growth[J]. Exp Cell Res, 2017,350(1):267-278.

[36] Li D J, Feng Z C, Li X R, et al. Involvement of methylation-associated silencing of formin 2 in colorectal carcinogenesis[J]. World J Gastroenterol, 2018,24(44):5013-5024.

[37] Jin J, Wang Y, Xu Y, et al. MicroRNA-144 regulates cancer cell proliferation and cell-cycle transition in acute lymphoblastic leukemia through the interaction of FMN2[J]. J Gene Med, 2017,19(6-7).

[38] Jin X, Wang J, Gao K, et al. Dysregulation of INF2-mediated mitochondrial fission in SPOP-mutated prostate cancer[J]. PLoS Genet, 2017,13(4):e1006748. 